\title{
Newton Iteration Method for Analysis of Suspension Cable
}

\author{
Jian Qin* and Yierta Ba \\ China Electric Power Research Institute, \\ Beijing, 100055, China \\ ${ }^{*}$ Corresponding author
}

\author{
Yan Ding \\ Risk Management Department, China Everbright Bank, \\ Beijing, 100032, China
}

\author{
Jieming Bai and Hongqiang Zhang \\ JiLin University, \\ Changchun, 130012, China
}

\begin{abstract}
A Newton iteration method is proposed based on the analytic method of segmental catenary for the suspension cable. The method is developed from the Newton iteration method for the nonlinear equations according to the analysis of different common methods. The tangent stiffness matrix of the iteration method is presented and the characteristics of the stiffness matrix are discussed. Then the iterative calculation process of suspension cable's form-finding for the specific engineering problem is listed. The method offers significant improvements in accuracy, efficiency and calculation amount of the solution, which can meet the needs of practical engineering. The results of the Newton iteration method are compared with the numerical results in other literatures and the contrast verifies the reliability of this method. The method is suitable for the analysis of suspension bridges, cargo ropeways and other various types of cable structures.
\end{abstract}

Keywords-suspension cable; catenary; stiffness matrix; iteration; form-finding

\section{INTRODUCTION}

Shape finding issue is first issue for analysis calculation of the cable structure. Because the cable has no stiffness (except tensile stiffness) itself and has no fixed form, imposed pretensile force and external load maintain balance through regulation of shape of the cable under given boundary conditions. Issue for determining initial balance shape of the cable is shape finding problem of the cable. There are mainly three methods for analysis of this issue: non-linear finite element method [1-3], force density method [4] and dynamic relaxation method [5], among of which non-linear finite element method is extensively applied in our country at present. The cable sections in these methods are mostly approximate shape, the obtained solution is mostly approximate solution.

Applying finite element method can solve complicate large engineering structure issue[6], but it isn't convenient for some specific issues. For example, the sectional suspension chain type is more convenient for shape finding analysis of main cable in the suspension cable bridge and loading calculation of the engineering goods transportation cable etc[7].
The sectional suspension chain method is commonly applied in finished bridge state of the suspension bridge, but corresponding calculation method has no great development in theory in recent 10 years, the circulation iteration method is mainly applied, variable is continuously adjusted until convergence of horizontal tensile force and height difference these two variables are finished. Application of this method is extensive, but calculation efficiency isn't high. This paper forwards the suspension chain Newton iteration method with main cable shape finding of the suspension cable and analysis of the engineering cable on basis of the sectional suspension chain method.

\section{ANALYTICAL CALCULATION OF SUSPENSION CHAIN}

\section{A. Basic Assumption}

Main cable is taken as ideal suspension cable according to suspension cable mechanical theory in analytic method, therefore following three basic assumptions are applied for main cable:

1) Main cable is ideal flexible cable; 2) Material of main cable is in accordance with Hook's Law; 3) Change quantity of section area and buck density of the main cable are very small which can be neglected.

\section{B. Basic Formula}

The cable section which only bears dead weight is shown in figure 1 . Take one fine section (figure 2) to carry out analysis.

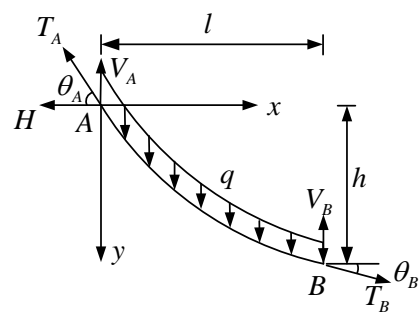

FIGURE I. FORCING ANALYSIS OF SUSPENSION CABLE 


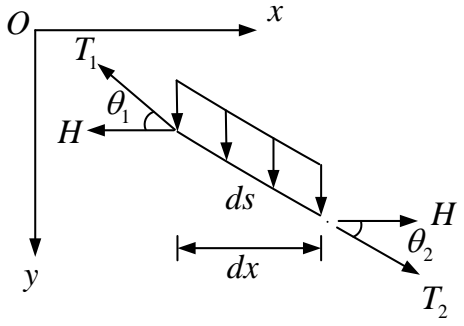

FIGURE II. SCHEMATIC FIGURE OF CABLE ELEMENT

Where, $T_{A}, T_{B}, V_{A}, V_{B}, \theta_{A}, \theta_{B}$ are cable tensile force, vertical component and angle at end point $\mathrm{A}$ and $\mathrm{B}$ respectively, $H$ is horizontal component of tensile force, $l$ and $h$ are horizontal distance between terminal ends and height difference.

The balance equation is

$$
y=\frac{H}{q} \cosh (a)-\frac{H}{q} \cosh \left(a-\frac{q}{H} x\right)
$$

Where, $q$ is dead load which is evenly distributed along arc, a is integral constant. Because of $\left.\frac{d y}{d x}\right|_{x=0}=\sinh (a)=\tan \theta_{A}=\frac{V_{A}}{H}$, and then $a=\sinh ^{-1}\left(V_{A} / H\right)$, which is determined by boundary conditions of the suspension cable.

\section{Balance Equation Of Suspension Cable Under Concentrated Loads}

Dead weight of the cable evenly distributed along arc and concentrated force transmitted by the cable are imposed on the main cable of the suspension cable bridge or good transportation cable (refer to figure 1). The suspension cable between hanging points only bears dead weight of the cable, therefore the whole suspension cable is considered as combination of multiple section suspension chain which is divided according to hanging points. For free cable status, it is simplified as flexible cable which only bears dead weight.

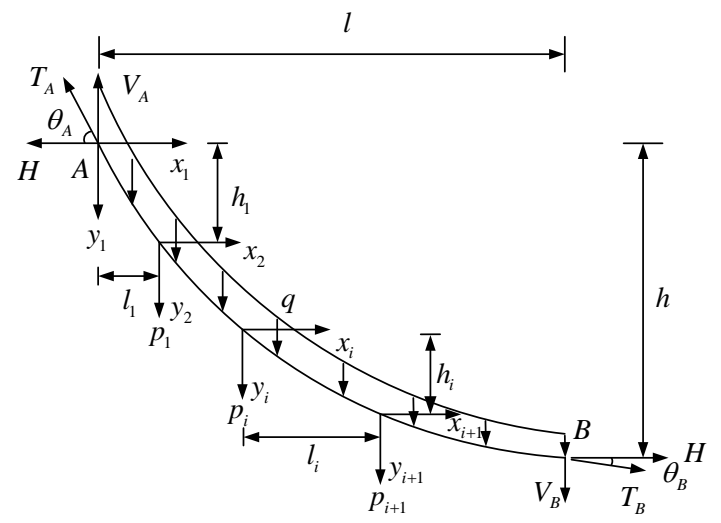

FIGURE III. THE CABLE SECTION ACTED BY GRAVITY AND CONCENTRATED LOADS
Assume the suspension cable only bears action of $n-1$ concentrated load $P_{i}(i=1,2, \ldots, n-1)$, the whole cable is divided into $\mathrm{n}$ sections, horizontal length is $l_{i}(i=1,2, \ldots, n)$ respectively.

Obtain balance equation of the whole cable at horizontal and vertical direction:

$$
H_{A}=H_{B}
$$

$$
V_{A}=q \sum_{i=1}^{n} S_{i}+\sum_{i=1}^{n-1} P_{i}+V_{B}
$$

There is compatibility equation of displacement

$$
\sum_{i=1}^{n} h_{i}=h
$$

Each cable section meets forcing balance condition at action point of concentrated load:

$$
H\left[\sinh \left(a_{i}-\frac{q}{H} l_{i}\right)-\sinh \left(a_{i+1}\right)\right]=P_{i}(i=1,2, \cdots, n-1)
$$

And then

$$
a_{1}=\sinh ^{-1}\left(\frac{V_{A}}{H}\right)
$$

$$
a_{i+1}=\sinh ^{-1}\left[\sinh \left(a_{i}-\frac{q}{H} l_{i}\right)-\frac{P_{i}}{H}\right]_{(i=1,2, \cdots, n-1)}
$$

\section{SHORTAGE OF CURRENT CALCULATION METHOD}

The current calculation method establishes recursion formula between each parameter $a_{i}$ according to the balance condition (1) at concentrated load acting point between each cable section, and then the initial condition is substituted into for calculation, repeatedly carry out iteration and correct the iteration value until the obtained results meet: 1) Compatibility condition of height difference (2); 2) suspension cable passes the appointed point. Shortcoming of the current method mainly has following two points:

- Because each parameter shall be obtained according to recursion formula during each iteration in the current iteration calculation process, and then calculate height of each cable section and carry out parameter correction, calculation efficiency is low, this recursion method can't realize parallel calculation in particular when large scale of calculations are related. 
- Because recursion formula is applied to calculate each parameter, the correction of each parameter is complicate and convergence speed of iteration is very slow.

\section{MATRIX ITERATION METHOD}

In order to realize extensive application of the model, the iteration correction algorithm with quick speed and high stability shall be researched, therefore this paper forwards quick Newton iteration method of the sectional suspension chain in the suspension chain theory.

\section{A. Calculation Of Initial Variable}

Boundary conditions of A point or B point shall be given during calculation, B point with low height is generally selected. Obtain two categories boundary conditions: 1) Slope at end point of suspension chain $\tan \theta_{B}$; 2) Vertical component force of end force $V_{B}$, relationship between them is $\tan \theta_{B}=\frac{V_{B}}{H}$.

When slope of the given end is $\tan \theta_{B}$, the approximate vector height $f$ is calculated by parabola formula, and then obtain initial cable length $S_{0}$, horizontal component force $H_{0}$ and end point vertical component force $V_{A 0}$ according to following formulas.

$$
\begin{gathered}
S_{0}=l\left(1+\frac{8 f^{2}}{3 l^{2}}\right) \\
H_{0}=\frac{q l^{2}}{8 f}+\frac{1}{h} \sum_{i=1}^{n}\left(P_{i} \sum_{j=1}^{i} l_{j}\right) \\
V_{A 0}=S_{0} q+\sum_{i=1}^{n-1} P_{i}+V_{B 0}=S_{0} q+\sum_{i=1}^{n-1} P_{i}+H_{0} \tan \theta_{B}
\end{gathered}
$$

Horizontal component force $H$ is obtained according to balance condition of torque.

Calculate and obtain initial parameter value $a_{i 0}(i=1,2, \ldots$, $n$ ) of each suspension cable by formula (3).

\section{B. Non-Linear Equation Group}

Let $t=H / q, p_{i}=P_{i} / q(i=1,2, \ldots, n-1)$, establish $n-1$ balance equations at vertical direction according to $n-1$ concentrated load:

$$
t\left[\sinh \left(a_{i-1}-\frac{l_{i-1}}{t}\right)-\sinh \left(a_{i}\right)\right]-p_{i}=0 \quad(\mathrm{i}=1,2, \ldots, \mathrm{n}-1)
$$

$$
\begin{aligned}
& t\left[\sinh \left(a_{n}-\frac{l_{n}}{t}\right)-\tan \theta_{B}\right]=0 \\
& \text { Or } t \sinh \left(a_{n}-\frac{l_{n}}{t}\right)-\frac{V_{B}}{q}=0
\end{aligned}
$$

And obtain compatibility equation (6) according to equation (5)

$$
t \sum_{i=1}^{n}\left[\cosh \left(a_{i}\right)-\cosh \left(a_{i}-\frac{l_{i}}{t}\right)\right]-h=0
$$

There are $n+1$ non-linear equations in total, unknown numbers are $t, a_{i}(i=1,2, \ldots, n)$, there are $n+1$ unknown numbers, i.e. this non-linear equation group is enclosed, it is expressed as:

$$
F(X)=0
$$

Where, $X=\left[a_{1}, a_{2}, \cdots, a_{n}, t\right]^{T}$. Use Newton iteration method to solve this non-linear equation.

\section{Non-Linear Tangent Stiffness Matrix}

It is necessary to calculate Jacobi matrix or value matrix when Newton iteration method is applied to solve. Expression of non-linear equation group is simple and clear in this issue, its Jacobi matrix is directly listed as following.

$$
F^{\prime}(X)=\frac{\partial F}{\partial X}=\left(\begin{array}{cc}
A_{n \times n} & B_{n \times 1} \\
C_{1 \times n} & D
\end{array}\right)_{(n+1) \times(n+1)}
$$

Where

$$
A=\left(\begin{array}{ccccc}
A_{11} & A_{12} & 0 & \ldots & 0 \\
0 & A_{22} & A_{23} & 0 & \vdots \\
\vdots & 0 & \ddots & \ddots & 0 \\
\vdots & & 0 & A_{n-1, n-1} & A_{n-1, n} \\
0 & \ldots & \cdots & 0 & A_{n, n}
\end{array}\right)_{n \times n}
$$

$$
A_{i, i}=t \cosh \left(a_{i}-\frac{l_{i}}{t}\right)_{(i=1,2, \cdots, n)}
$$

$$
A_{i, i+1}=-t \cosh \left(a_{i}\right)(i=1,2, \cdots, n-1)
$$

$$
B_{i}=\sinh \left(a_{i}-\frac{l_{i}}{t}\right)-\sinh \left(a_{i+1}\right)+\frac{l_{i}}{t} \cosh \left(a_{i}-\frac{l_{i}}{t}\right)
$$

And boundary equation 


$$
\begin{gathered}
(i=1,2, \cdots, n-1) \\
B_{n}=\sinh \left(a_{n}-\frac{l_{n}}{t}\right)-\tan \theta_{B}+\frac{l_{n}}{t} \cosh \left(a_{n}-\frac{l_{n}}{t}\right) \\
B_{n}=\sinh \left(a_{n}-\frac{l_{n}}{t}\right)+\frac{l_{n}}{t} \cosh \left(a_{n}-\frac{l_{n}}{t}\right) \\
C_{i}=t \sinh \left(a_{i}\right)-t \sinh \left(a_{i}-\frac{l_{i}}{t}\right)(i=1,2, \cdots, n) \\
D=\sum_{i=1}^{n}\left[\cosh \left(a_{i}\right)-\cosh \left(a_{i}-\frac{l_{i}}{t}\right)-\frac{l_{i}}{t} \sinh \left(a_{i}-\frac{l_{i}}{t}\right)\right]
\end{gathered}
$$

Most elements in this matrix are zero, the sub-matrix A is simple upper triangular matrix and there is non-zero element on the diagonal line and secondary diagonal line, therefore elimination method can be applied in the reverse matrix for calculation of the tangent stiffness matrix.

\section{Calculation}

After the tangent stiffness matrix is obtained, result can be calculated by Newton iteration method for the non-linear equation group. Firstly obtain initial iteration value $X_{0}$ according to calculation for this issue, and then carry out iteration according to following formula

$$
X_{n+1}=X_{n}+\left[F^{\prime}\left(X_{n}\right)\right]^{-1} \cdot F\left(X_{n}\right)(n=0,1,2, \cdots)
$$

Because structure of Jacobi matrix $F^{\prime}\left(X_{n}\right)$ in non-linear equation group for this issue is regular, non-zero element is less, calculation quantity for solving inverse is less, so calculation efficiency of Newton iteration method is higher than that of intended Newton method or corrected Newton method. Convergence speed of this method is quick, it is generally convergent to accuracy of $10^{-8}$ after $4 \sim 8$ times iteration.

\section{ANALYSIS OF UNDETERMINED PROBLEM}

The suspension cable shall pass the appointed point $\left(O_{x}, O_{y}\right)$ in some engineering issue, search slope $\tan \theta_{B}$ of the end point in the boundary condition, judge whether obtained suspension cable curve from calculation passes through the appointed point until it meets accuracy requirement, the calculation process figure is shown as figure 4 .

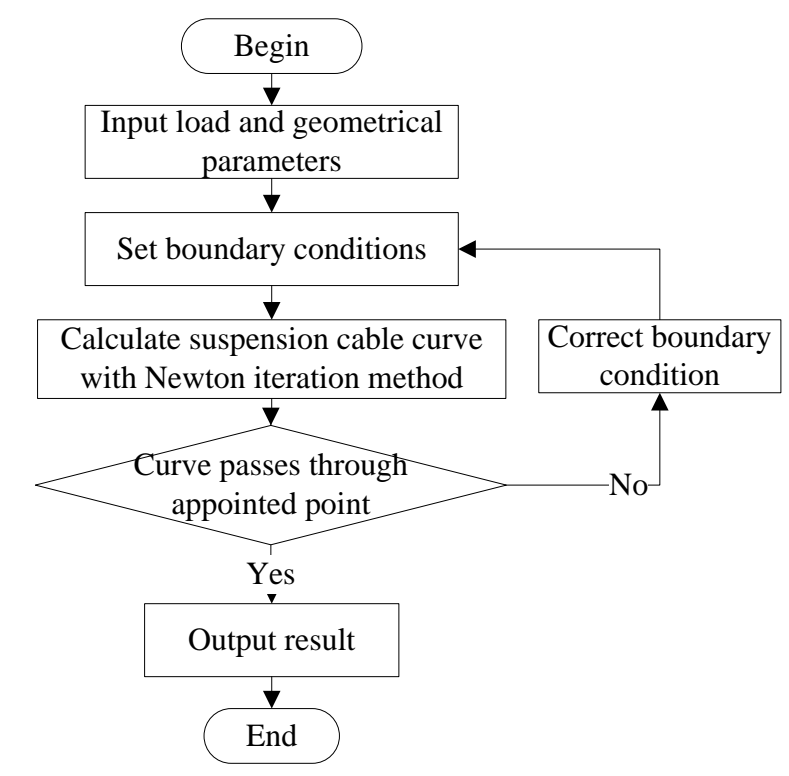

FIGURE IV. THE CALCULATION PROCESS OF SUSPENSION CABLE

\section{CALCULATION SAMPLE}

This paper establishes the whole bridge model of the common vertical suspension cable bridge in paper [1]. The span is $888 \mathrm{~m}$, and there are 37 concentrated loads $(q=50,100$, $200 \mathrm{kN} / \mathrm{m})$. The density of cable is $54000 \mathrm{~N} / \mathrm{m}$, the $f=60,80$ and $100 \mathrm{~m}$.

The $H$ and displacement $\left.y\right|_{x_{0}}$ at $x_{0}=216 \mathrm{~m}$ are shown in Table 1.

TABLE I. THE RESULTS COMPARISON BETWEEN NEWTON METHOD AND METHOD FROM REFERENCE

\begin{tabular}{cccccccc}
\hline \multicolumn{2}{c}{ Case } & \multicolumn{3}{c}{$H(\mathrm{kN})$} & & \multicolumn{3}{c}{$\left.y\right|_{x_{0}}(\mathrm{~m})$} & \\
\hline$q$ & $f$ & Reference & Eesult & Error & Reference & Eesult & Error \\
\hline 0 & 60 & 89246.7 & 89246.0 & 0.66 & 44.2484 & 44.248 & 0.000 \\
& & & & & & 0 & 3 \\
& 60 & 171386.2 & 171386.7 & 0.49 & 44.2145 & 44.214 & 0.000 \\
& & & 128847.8 & & & 6 & 1 \\
50 & 80 & 128845.9 & 3 & 1.93 & 58.9905 & 58.989 & 0.000 \\
& 10 & & & & & 7 & 7 \\
& 0 & 103390.7 & 103390.4 & 0.31 & 73.7950 & 73.795 & 0.000 \\
& 60 & 253529.2 & 253526.9 & 2.24 & 44.2023 & 44.202 & 0.000 \\
10 & & & & & & 8 & 5 \\
0 & 80 & 190454.4 & 190453.4 & 1.00 & 58.9617 & 58.962 & 0.000 \\
& 10 & & & & & 1 & 4 \\
& 0 & 152675.7 & 152675.4 & 0.26 & 73.7418 & 73.742 & 0.000 \\
& & & & & & 0 & 2 \\
& 60 & 417801.2 & 417807.2 & 5.97 & 44.1937 & 44.193 & 0.000 \\
20 & & & & & & 1 & 5 \\
0 & 80 & 313663.4 & 313663.8 & 0.46 & 58.9394 & 58.939 & 0.000 \\
& 10 & & & & & 4 & 0 \\
& 0 & 251245.7 & 251244.3 & 1.38 & 73.6977 & 73.698 & 0.000 \\
& & & & & & 5 \\
\hline
\end{tabular}


It is found that the error between reference and result of method in this paper is very small, relative value of the free stress cable length at left and right span are obtained with the same method respectively, which are $1.2 \mathrm{e}-5$ and $7.3 \mathrm{e}-6$ respectively, therefore it is said that this program can ensure accuracy of the result obtained for the free stress cable length of the suspension bridge.

\section{CONCLUSIONS}

Main cable is main bearing member of the suspension cable bridge, it is very important to accurately find shape of its finished bridge. Whether shape of the main cable is correct or not, it directly affects whether blanking length of the main cable, installation position of each cable clamp and length of the corresponding suspension cable etc parameters are correct or not.

The analytic iteration method is proposed for analysis of suspension and the form of the tangent stiffness matrix is given. The corresponding iterative calculation process is also given for specific engineering problems. The method proposed in this paper is more advanced than the traditional method, and it has good precision and is suitable for the calculation of various forms of suspension bridges, freight transportation and so on.

By comparing with other documents, it can be seen that the results obtained by this method can meet the practical engineering needs.

\section{REFERENCES}

[1] Shen Ruili. Calculation methods for design and erection of cable curve of suspension bridge [J]. China Civil Engineering Journal, 1996, 29(2): 3 9.

[2] Zhang Zhiguo, Jin Mingjun, Zou Zhenzhu. Static solution of suspension cables under tare load [J]. China Railway Science, 2004, 25(3): 67 70.

[3] Ociisendorp J A, Billington D P. Self-anchored suspension bridges [J]. Journal of Bridge Engineer, 1999(8): 151 156.

[4] Yuan Xingfei, Dong Shilin. A two-node curved cable element for nonlinear analysis [J]. Engineering Mechanics, 1999, 16(4): 59 64.

[5] Yang Menggang, Chen Zhengqing. Nonlinear analysis of cable structure using a two-node curved cable element of high precision [J]. Engineering Mechanics, 2003, 20(1):42 47.

[6] Bai Xuesong, Miao Qian. Research on calculation model based on suspension cable theories in construction cargo cableway [J]. Power System Technology, 2008, 32(20): 90 94.

[7] Tang Maolin, Qiang Shizhong, Shen Ruili. Segmental catenary method of calculating the cable curve of suspension bridge [J]. China Railway Science, 2003, 25(1): 87 91. 